\title{
Pour en lire plus : Le Débat fertile. Explorer une controverse dans l'émotion.
}

Jean-Claude Guerrini

\section{OpenEdition}

1 Journals

Édition électronique

URL : https://journals.openedition.org/ere/7529

ISSN : 2561-2271

Éditeur

Centr'ERE

Référence électronique

Jean-Claude Guerrini, «Pour en lire plus : Le Débat fertile. Explorer une controverse dans l'émotion. », Éducation relative à l'environnement [En ligne], Volume 17-1 | 2022, mis en ligne le 27 janvier 2022, consulté le 02 février 2022. URL : http://journals.openedition.org/ere/7529

Ce document a été généré automatiquement le 2 février 2022

La revue Éducation relative à l'environnement est mise à disposition selon les termes de la Licence Creative Commons Attribution - Pas d'Utilisation Commerciale 4.0 International. 
Pour en lire plus : Le Débat fertile. Explorer une controverse dans l'émotion. Jean-Claude Guerrini

RÉFÉRENCE

Claire Polo (2020). Le Débat fertile. Explorer une controverse dans l'émotion. Grenoble : UGA Éditions, 260 pages 


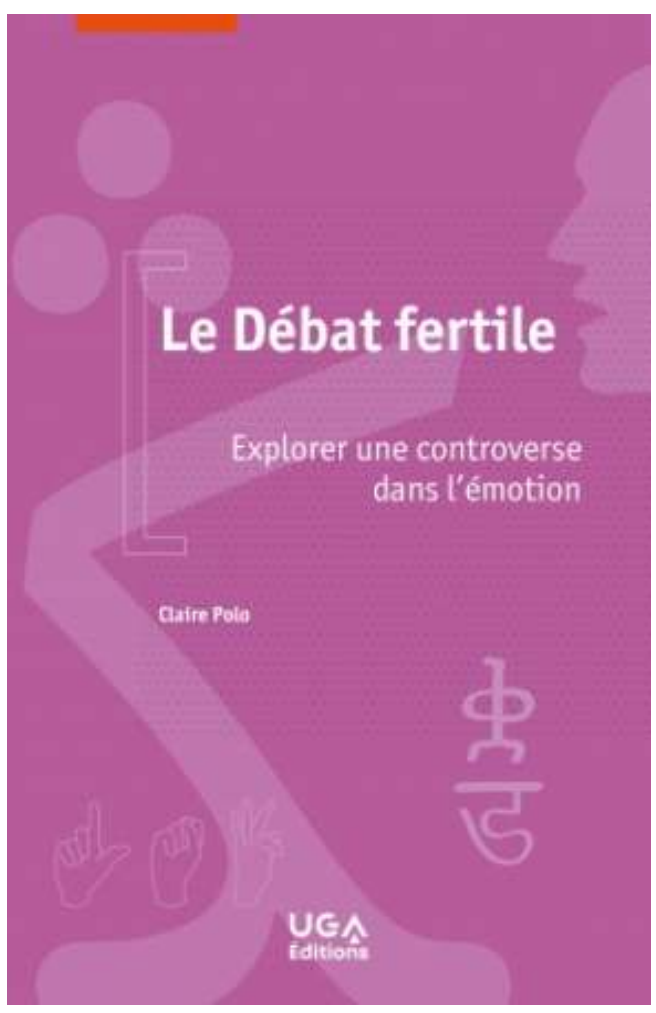

Dans cet ouvrage publié dans la collection «Langues, Gestes, Paroles » (UGA Éditions), Claire Polo, Maîtresse de conférences en Sciences de l'éducation à l'Université Lyon 2, se donne un double objectif : communiquer son intérêt pour une conception dialogique de l'activité argumentative sensible à la dimension émotionnelle des échanges et partager les outils conceptuels et didactiques qu'elle a forgés dans sa pratique d'animatrice d'ateliers d'éducation populaire, d'enseignante et de chercheuse.

Dès sa formation à Sciences Po Grenoble, l'auteure était soucieuse de distinguer un débat "stérile » d'un débat "fertile ", afin de saisir en profondeur les enjeux d'une controverse et d'alimenter la construction collective d'arguments mieux fondés, sinon convergents. Elle a ainsi identifié dans les controverses socioscientifiques portant sur des questions complexes et socialement vives (progrès du génie génétique, potentialités des nanotechnologies, réchauffement climatique, épuisement des ressources, etc.), un objet privilégié pour mettre au jour les conditions de possibilité d'une pratique argumentative constructive menée collectivement.

3 Confrontée à la dimension émotionnelle des échanges que ces controverses suscitaient, loin d'y voir un obstacle ou une menace dont il serait impératif de se prémunir, Claire Polo a choisi de la soumettre à un examen méthodique. À l'issue de son travail de thèse (L'eau à la bouche. Ressources et travail argumentatifs des élèves lors de débats socioscientifiques sur l'eau potable, 2014) et contrairement à l'idée généralement admise selon laquelle présenter « un argument comme fondé sur une logique affective serait prêter le flanc à une accusation de raisonnement irrationnel et fallacieux» (p.240), elle s'estime aujourd'hui en mesure d'affirmer que la connaissance et le contrôle de la tonalité émotionnelle d'un débat contribue à "favoriser une exploration constructive » de ses enjeux (p. 51).

4 Cette conviction résulte d'une enquête empirique extrêmement ambitieuse, éclairée par la prise en compte de nombreuses disciplines (didactique des sciences, linguistique, 
étude des interactions communicatives, études d'argumentation) et menée grâce à la mobilisation d'un dispositif didactique original baptisé YouTalk dans des contextes pédagogiques variés, en France, au Mexique et aux Etats-Unis.

5 Élaboré initialement dans le cadre d'un partenariat entre le laboratoire interdisciplinaire ICAR (Interactions, Corpus, Apprentissages, Représentations, UMR 5191) et l'antenne lyonnaise de l'association de médiation scientifique Petits Débrouillards, le dispositif YouTalk a été conçu pour permettre à des jeunes élèves de 12 à 15 ans de débattre de controverses socioscientifiques, non pas sous la conduite de leurs professeurs, mais sous la forme d'une activité éducative fortement structurée d'environ deux heures animée par un binôme d'élèves volontaires plus âgé(e)s (16 à 19 ans). Après une brève introduction destinée à familiariser les élèves avec la thématique retenue, l'activité, qui alterne des phases en classe entière et en petits groupes de 3 ou 4 élèves, recourt, pour guider les élèves dans leur réflexion, à un questionnaire à choix multiples (QCM) projeté à l'écran. Par exemple, pour la question principale (QP) qui leur est soumise (" À ton avis, l'accès d'une personne à l'eau potable, à l'avenir dépendra surtout... ?), ils doivent choisir entre les items suivants : a) de sa richesse ; b) de sa capacité physique à vivre avec une eau de moindre qualité ; c) des efforts faits dès maintenant pour économiser l'eau et la préserver; d) de l'endroit où cette personne naîtra sur la planète ; e) de la capacité de la nature à s'adapter à nos besoins en eau ;f) des avancées scientifiques). » Après avoir répondu individuellement et anonymement à cette question, les élèves doivent se prononcer, lors de 3 sessions thématiques, sur deux séries de questions dont le statut épistémique est fermement distingué : des questions de connaissance $(\mathrm{QC})$ pour lesquelles on attend la «bonne réponse » (qui sera fournie par une diapo explicative), puis des questions d'opinion (QO) pour lesquelles des options diverses sont proposées. Claire Polo estime en effet qu'il est indispensable de sensibiliser les élèves à « la distinction entre faits et hypothèses vérifiables d'une part, opinions et croyances d'autre part» (p.32). Les questions de connaissance sont l'occasion de fournir des informations permettant au débat de se développer sur des bases scientifiques solides.

6 L'examen des questions d'opinion, que les concepteurs de la séance doivent élaborer de manière à favoriser au maximum l'exploration de la question principale, vise plusieurs objectifs : les élèves doivent s'entraîner à s'approprier un sujet nouveau et complexe, à se forger une opinion, à travailler en groupe et à prendre la parole en public. Grâce à un système de vote électronique, les animateurs recueillent anonymement, outre les réponses initiale et finale à la question principale, la réponse aux questions de connaissance qui donnent lieu dans chaque groupe à une brève discussion. Tout au long de la séance, les sous-groupes sont appelés à se prononcer collectivement sur les questions d'opinion à la suite d'un échange d'une durée suffisante pour permettre d'évaluer la pertinence des options proposées. Il n'y a pas de porte-parole, mais lors de la mise en commun, les élèves peuvent manifester leur désaccord avec le choix effectué au sein de leur sous-groupe. La synthèse assurée par un animateur ou une animatrice débouche, après une nouvelle discussion dans les groupes, sur le vote individuel et anonyme final.

7 En filmant ces « cafés scientifiques » (22 séquences, dont une dizaine analysée en détail pour sa thèse), Claire Polo a collecté des données (textuelles et mimo-gestuelles) très abondantes dont plusieurs transcriptions commentées dans l'ouvrage permettent de mesurer la qualité des interprétations auxquelles elles ont donné lieu. La dimension 
socioaffective de ces débats, qu'elle tient constamment à associer aux objectifs descriptifs et prescriptifs poursuivis par les élèves, est examinée à travers les catégories psychologiques du thymique et du phasique (Plantin, 2011) qui renvoient, respectivement, à la tonalité affective de fond, relativement stable, des échanges et à leurs épisodes émotionnels de plus grande intensité. À côté des émotions argumentées, explicitement thématisées, Polo s'intéresse aux autres modes de sémiotisation de l'émotion, qu'il s'agisse des émotions explicitement argumentées, des émotions implicites (montrées ou induites) ou des "paramètres émotionnants", ces "objets d'accord» affectifs culturellement partagés qui constituent des ressources pour argumenter (Micheli, 2010).

8 S'inspirant des travaux de didactique de Wegerif et Mercer (1997) qui soulignent la supériorité du " discours d'exploration ", par rapport à « l'accumulation d'idées » ou à la «dispute» dans l'apprentissage en petits groupes, Claire Polo examine la manière dont ces trois types de discours interviennent dans la dynamique des cafés scientifiques en favorisant, selon les cas, des comportements consensuels, compétitifs ou de critique constructive. En promouvant le «discours d'exploration » investi émotionnellement, qui ouvre un espace de débat où les options concurrentes peuvent être présentées «sans danger pour les faces qui les portent» (p.163), l'autrice, qui s'inscrit dans le courant actuel de réhabilitation de certains aspects de la rhétorique classique, se démarque nettement des critiques normatives de la fin du $\mathrm{XX}^{\mathrm{e}}$ siècle hostiles à la prise en compte des émotions au nom d'une dichotomie irrecevable raison/émotion.

9 La dimension comparative de ce travail qui suggère l'existence d'approches argumentatives distinctes selon les terrains d'observation étasunien, mexicain et français ne peut être abordée - Polo en convient - qu'avec beaucoup de prudence, compte tenu du caractère limité de l'étude (p. 200). Mais nous disposons avec cet ouvrage extrêmement stimulant d'un outil d'une remarquable précision, non seulement pour des usages didactiques ou pour l'animation d'ateliers de réflexion citoyens, mais aussi pour la conduite d'études comparatives de grande ampleur.

\section{BIBLIOGRAPHIE}

Micheli, R. (2010) L'émotion argumentée : l'abolition de la peine de mort dans le débat parlementaire français. Paris : Cerf.

Plantin, C. (2011). Les bonnes raisons des émotions : principes et méthode pour l'étude du discours "émotionné ». New York : Peter Lang.

Wegerif, R. et Mercer, N. (1997). A dialogical framework for researching peer talk. Language and Education Library, 12, 49-64. 
AUTEUR

JEAN-CLAUDE GUERRINI

Université de Lyon 